\title{
Síntese e Caracterização de Nanocompósitos Esfoliados de Poliestireno - Hidróxido Duplo Lamelar Via Polimerização In Situ
}

\author{
Rodrigo Botan, Telma R. Nogueira, Liliane M. F. Lona \\ Departamento de Processos Químicos, UNICAMP
}

\author{
Fernando Wypych \\ Departamento de Química, UFPR
}

\begin{abstract}
Resumo: Nanocompósitos Esfoliados de Poliestireno (PS) e Hidróxido Duplo Lamelar (HDL) composto por zinco, alumínio e dodecil sulfato de sódio, como ânion interlamelar, foram sintetizados via polimerização in situ. O efeito de diferentes composições de HDL $(0,5,1,3$ e $5 \%$ em massa) foi avaliado. Os nanocompósitos obtidos foram caracterizados através de ensaios de Difração de Raios X (DRX), Espectroscopia no Infravermelho por Transformada de Fourier (FTIR), Microscopia Eletrônica de Transmissão (MET) e Análise Termogravimétrica (TGA). Os resultados obtidos através das análises de DRX e MET mostraram que todos os nanocompósitos produzidos apresentaram morfologia esfoliada. Os resultados das TGA, quando se utilizam 50\% de perda de peso como ponto para comparação, apresentaram ganhos significativos de estabilidade térmica para todas as composições dos nanocompósitos em comparação ao poliestireno puro. Este comportamento possibilita uma vasta gama de aplicação destes novos materiais em diversos campos da indústria e pesquisa.
\end{abstract}

Palavras-chave: Nanocompósito, hidróxido duplo lamelar, poliestireno, polimerização in situ.

\section{Synthesis and Characterization of Exfoliated Polystyrene-Layered Double Hydroxide Nanocomposites Via In Situ Polymerization}

\begin{abstract}
Exfoliated nanocomposites of polystyrene (PS) and layered double hydroxide (LDH) compound of zinc, aluminium and sodium dodecyl sulfate, as interlayer anion, have been synthesized via in situ polymerization. The effect from the LDH compositions $(0.5,1,3,5 \%$ weight ) was studied. The nanocomposites were characterized by X-ray Diffraction (XRD), Fourier Transform Infrared Spectroscopy (FTIR), Transmission Electron Microscopy (TEM) and Thermogravimetric Analysis (TGA). The XRD and TEM results showed an exfoliated morphology for the nanocomposites in all compositions. With the 50\% weight loss taken as a reference, the TGA results indicated significant improvement in thermal stability for all compositions of the nanocomposites, in comparison to pure polystyrene. This is promising for the application of these new materials in many fields of industry and research.
\end{abstract}

Keywords: Nanocomposite, layered double hydroxide, polystyrene, in situ polymerization.

\section{Introdução}

Os nanocompósitos podem ser definidos como materiais híbridos em que pelo menos um dos componentes tem dimensões nanométricas ${ }^{[1-3]}$. Nanocompósitos poliméricos apresentam recente desenvolvimento e têm como marco inicial a publicação de um trabalho na década de 80/90 pelo grupo de pesquisa da empresa Toyota, onde foi produzido um nanocompósito de nylon 6 e o argilomineral (montimorilonita). O novo nanocompósito produzido apresentou uma extraordinária melhora nas suas propriedades mecânicas, térmicas e físicas quando comparado ao polímero puro ${ }^{[4]}$.

Entre os nanocompósitos poliméricos, muitos estudos mostram como a dispersão de compostos lamelares em uma matriz polimérica afeta as propriedades do material obtido, no entanto, na maioria dos trabalhos são utilizados argilominerais trocadores catiônicos naturais, como a montmorilonita ${ }^{[2,3]}$. Outros materiais lamelares naturais e sintéticos, como os hidróxidos duplos lamelares (HDL's), apesar de potencialmente interessante, têm sido pouco explorados.

Os HDL's são uma classe emergente de compostos lamelares naturais ou sintéticos, com grande potencial para utilização como reforço em nanocompósitos de matriz polimérica. Estes materiais apresentam como composição química $\left[\mathrm{M}_{1-\mathrm{x}}^{\mathrm{II}} \mathrm{M}_{\mathrm{x}}^{\mathrm{III}}(\mathrm{OH})_{2}\right]$ $\left[\mathrm{A}^{\mathrm{n}-}{ }_{\mathrm{x} / \mathrm{n}} \cdot m \mathrm{H}_{2} \mathrm{O}\right]$, onde $\mathrm{M}^{\mathrm{II}}$ representa um cátion divalente, $\mathrm{M}^{\mathrm{III}}$ representa um cátion trivalente, $\mathrm{A}^{\mathrm{n}-}$ representa um ânion n-valente (por exemplo $\mathrm{Cl}^{-}, \mathrm{NO}_{3}^{-}, \mathrm{SO}_{4}^{2-}, \mathrm{CO}_{3}$, etc) e $m$ representa o número de moléculas de água ${ }^{[5-9]}$. Os HDL's apresentam uma estrutura semelhante à da brucita $\left(\mathrm{Mg}(\mathrm{OH})_{2}\right)$, podem ser sintetizados por meio de rotas relativamente simples a um custo relativamente baixo, com controle de tamanho e uniformidade na distribuição de tamanho das partículas.

Para a produção dos nanocompósitos existem algumas rotas já conhecidas e a polimerização in situ é particularmente interessante, uma vez que possibilita uma distribuição mais homogênea do reforço inorgânico na matriz orgânica (em um polímero fundido, a distribuição do reforço não é homogênea devido à alta viscosidade do meio e também as agregações das partículas nanométricas são difíceis de serem evitadas), o que é de fundamental importância para a preparação de materiais de alta qualidade ${ }^{[10]}$.

Para produção de nanocompósitos poliméricos (poliestireno) com HDL deve-se considerar a incompatibilidade entre estes dois materiais, pois os HDL's apresentam predominantemente uma natureza hidrofílica enquanto o polímero (poliestireno) apresenta uma natureza predominantemente hidrofóbica. Portanto para produção destes nanocompósitos faz-se necessária a modificação química das superfícies destes HDL's de forma a torná-los hidrofóbicos. Normalmente são utilizados agentes que 
promovem a compatibilização química por intermédio de ligações de hidrogênio, interações eletrostáticas ou por ligações covalentes na interface inorgânica/orgânica (funcionalização).

O principal objetivo na síntese de nanocompósitos é a preparação de materiais de alta qualidade através de uma distribuição uniforme das cargas na matriz polimérica e uma boa adesão na interface dos dois componentes, para que assim ocorra um sinergismo entre matriz e reforço. Este trabalho descreve a síntese e caracterização de nanocompósitos esfoliados de poliestireno/ hidróxido duplo lamelar ( $\mathrm{ZnAl})$ modificados com dodecil sulfato de sódio (DDS) em quatro composições distintas via polimerização in situ utilizando a técnica de polimerização em massa.

\section{Experimental}

\section{Materiais}

Os materiais utilizados para síntese do hidróxido duplo lamelar foram dodecil sulfato de sódio (DDS) $\left(\mathrm{C}_{12} \mathrm{H}_{25} \mathrm{SO}_{4} \mathrm{Na}\right)$ (Synth), cloreto de zinco $\left(\mathrm{ZnCl}_{2}\right)$ e cloreto de alumínio $\left(\mathrm{AlCl}_{3}\right)$ (Ecibra) e hidróxido de sódio ( $\mathrm{NaOH})$ (Fmaia).

Para síntese dos nanocompósitos in situ foram utilizados hidróxido de sódio $(\mathrm{NaOH})$ (Fmaia), cloreto de cálcio $\left(\mathrm{CaCl}_{2}\right)$ (Ecibra), monômero (estireno) com 99\% de pureza (Sigma Aldrich) e o iniciador Tert - Butilperóxido-2-Etilhexil Carbonato (TBEC) (Sigma - Aldrich).

\section{Métodos}

O HDL foi sintetizado pelo método de co-precipitação ${ }^{[11]}$. Neste trabalho foi produzido HDL com a composição química nominal de $\mathrm{Zn}_{0,66} \mathrm{Al}_{0,33}(\mathrm{OH})_{2}(\mathrm{DDS})_{0,33} \mathrm{nH}_{2} \mathrm{O}$.

$\mathrm{Na}$ síntese do HDL foram adicionados ao reator DDS e água deionizada. Estes reagentes ficaram em agitação durante quinze minutos. Logo após foi adicionada lentamente ao reator (gota a gota) uma solução de sais, composta por cloreto de zinco e cloreto de alumínio. Simultaneamente, adicionou-se uma solução básica formada por água deionizada e hidróxido de sódio, com o objetivo de controlar o $\mathrm{pH}$ do meio reacional de tal forma que ele ficasse em torno de 10 . Os reagentes permaneceram em agitação constante durante sete horas para a cristalização e crescimento dos cristais do HDL. Todo esse processo de síntese também foi acompanhado por um controle de $\mathrm{pH}$, temperatura $\left(35^{\circ} \mathrm{C}\right)$ e atmosfera de nitrogênio para manter o sistema inerte.

Para síntese dos nanocompósitos foi utilizada a polimerização em massa via radical livre. Neste trabalho foram sintetizados o poliestireno puro e os nanocompósitos, sendo o nanocompósito formado por poliestireno/ hidróxido duplo lamelar modificado com DDS (PS - $\mathrm{Zn}_{0,66} \mathrm{Al}_{0,33}(\mathrm{OH})_{2}(\mathrm{DDS})_{0,33} \mathrm{nH}_{2} \mathrm{O}$ ). Foram produzidos nanocompósitos com diferentes percentuais de carga inorgânica (HDL) $(0,5 ; 1 ; 3$ e $5 \%$ em massa).

A primeira tarefa realizada antes do procedimento experimental de polimerização é a purificação do monômero. O monômero foi lavado por três vezes com uma solução de $\mathrm{NaOH}$ e três vezes com água deionizada. Depois de lavado, este monômero foi seco com $\mathrm{CaCl}_{2}$.

Para a polimerização do poliestireno puro foram utilizados monômero e iniciador e para os nanocompósitos foram utilizados o mesmo monômero e iniciador com o acréscimo do HDL.

As reações de polimerização em massa para a produção do polímero puro $^{[12]}$ e dos nanocompósitos foram realizadas em ampolas e ocorreram em um regime de batelada a uma temperatura de $125{ }^{\circ} \mathrm{C}$ durante um período de quatro horas em banho com circulação de fluido. Nestas reações o iniciador TBEC foi utilizado na concentração de 0,0029 mol. $\mathrm{L}^{-1}$.

\section{Caracterização}

Para caracterização dos materiais deste trabalho foram utilizadas as técnicas de DRX, através de um equipamento da marca Shimadzu, modelo XRD 7000. Este equipamento utilizou um cátodo de cobre com comprimento de onda $1,5406 \AA$, potência da fonte de $40 \mathrm{kV}$ e corrente de $30 \mathrm{~mA}$. A velocidade de varredura utilizada foi de $0,02^{\circ}$ a cada 30 segundos e a faixa varrida foi de (20) 1,5 até $70^{\circ}$.

Para o FTIR foi utilizado um equipamento da marca Perkin Elmer, com leitura na região de $4.000 \mathrm{a} 400 \mathrm{~cm}^{-1}$, esta análise foi realizada utilizando-se pastilha de $\mathrm{KBr}$ na proporção de $1 \mathrm{mg}$ de amostra para $100 \mathrm{mg}$ de $\mathrm{KBr}$. Na análise do MET foi utilizado o modelo JEM 3010, da marca Joel com uma aceleração de voltagem de $300 \mathrm{kV}$. As amostras utilizadas nesta análise apresentavam uma espessura de $120 \mathrm{~nm}$ e foram cortadas em um ultramicrótomo a temperatura ambiente com faca de vidro.

As medidas de TGA foram realizadas no equipamento da marca Universal, modelo V2.3. Neste ensaio foi utilizada atmosfera de nitrogênio com fluxo de $100 \mathrm{~mL} / \mathrm{min}$ e taxa de aquecimento de $10{ }^{\circ} \mathrm{C} / \mathrm{min}$. Utilizou-se uma faixa de aquecimento da temperatura ambiente de 25 até $600{ }^{\circ} \mathrm{C}$.

\section{Resultados e Discussão}

\section{Análise de DRX}

A análise de DRX foi realizada no HDL sintetizado de composição química nominal $\mathrm{Zn}_{0,66} \mathrm{Al}_{0,33}(\mathrm{OH})_{2}(\mathrm{DS})_{0,33} \mathrm{nH}_{2} \mathrm{O}$, no poliestireno puro e nos nanocompósitos nas quatro composições consideradas (Figura 1).

Através do difratograma é possível verificar e confirmar a cristalinidade e a estrutura lamelar do HDL produzido, com a observação de uma série de picos basais, característico para esse tipo de composto. Com os picos basais deste HDL, foi calculado o seu espaçamento basal (d). Para este cálculo, utilizaram-se os dados do difratograma através do pico de maior ordem possível e mais característico do HDL e a equação de Bragg (n $\lambda=2 \mathrm{~d} \operatorname{sen} \theta)$. Assim com este cálculo foi possível constatar que o HDL sintetizado apresentou um espaçamento basal (d) de $26,59 \AA$, o que concorda com a literatura ${ }^{[13,14]}$. A partir deste valor de espaçamento basal, retirando o valor da lamela de 4,8 $\AA$ (valor da lamela igual ao da brucita, a qual possui o valor conhecido e presente na literatura), é possível obter o valor do espaçamento interlamelar, o qual é de 21,79 Å para o HDL sintetizado.

O valor do espaçamento interlamelar, retirando o valor da lamela da brucita $(4,8 \AA$ ) para a hidrotalcita é de 2,8 a 3,0 ̊̊ podendo variar dependendo do grau de hidratação do material ${ }^{[13-14]}$. Portanto através destes valores fica comprovado que o HDL sintetizado realmente sofreu uma modificação e, como consequência desta modificação, ocorreu o aumento do espaçamento interlamelar, uma vez que o resultado obtido $(21,79 \AA)$ é consideravelmente maior do que os referenciados para um HDL sem modificação (hidrotalcita).

Com o aumento do espaçamento interlamelar, as forças que mantêm as lamelas do HDL unidas diminuem, permitindo a intercalação de compostos orgânicos neste domínio, o que possibilita que este composto orgânico, neste caso um monômero, quando polimerizado junto com o HDL, delamine a estrutura do HDL e forme o nanocompósito ${ }^{[3,15]}$.

Feita a caracterização por DRX para o HDL, foi feito o mesmo ensaio para o poliestireno puro e nanocompósitos.

O poliestireno puro sendo um material amorfo como é observado na Figura 1 não apresenta nenhum tipo de pico característico na DRX, apenas pequenos halos amorfos, uma vez que não possui nenhuma estrutura cristalina de longo alcance. 


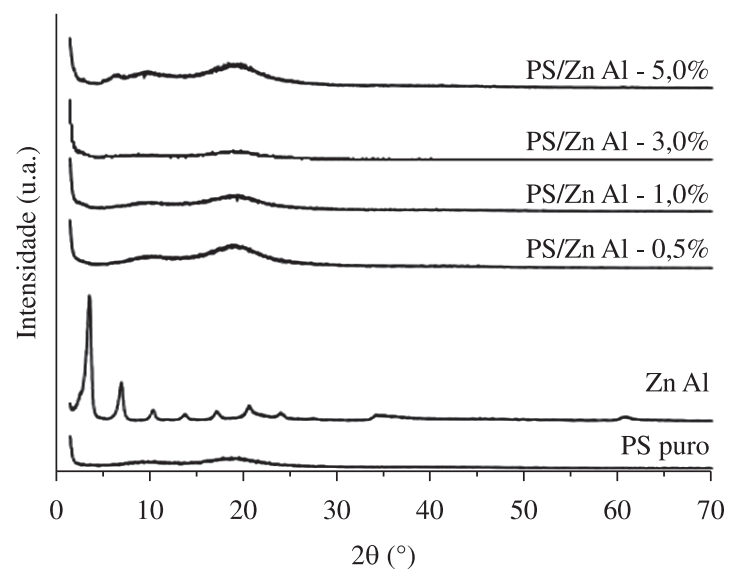

Figura 1. Difratogramas de raios $\mathrm{X}$ do $\mathrm{HDL}(\mathrm{ZnAl})$, PS puro e nanocompósitos formados por PS/ ZnAl.

Os difratogramas para os nanocompósitos mostrados na Figura 1 nas composições de $0,5,1,3$ e $5 \%$, demonstraram que todos os nanocompósitos sintetizados apresentaram seus difratogramas iguais ou muito próximos ao do poliestireno puro, sem nenhum pico que caracterizasse uma estrutura cristalina de longo alcance, apresentando apenas pequenos halos, os quais também são observados no difratograma do poliestireno puro.

Sendo assim, com os difratogramas destes nanocompósitos iguais ou muito próximos ao do poliestireno puro, e não existindo picos que caracterizem algum tipo de estrutura cristalina (empilhamento das lamelas) do HDL na matriz polimérica, conclui-se que estes nanocompósitos apresentam uma estrutura sem cristalinidade. Com estes resultados, é possível explanar que a estrutura do HDL foi "quebrada", ou seja, as lamelas do HDL foram delaminadas dentro da matriz polimérica, o que caracteriza e sugere fortemente um nanocompósito com estrutura ou morfologia esfoliada.

Nesta estrutura (esfoliada) ocorre a completa delaminação do HDL, ficando suas lamelas distribuídas aleatoriamente dentro da matriz polimérica em escala nanométrica. Estas monolamelas com nanômetros criam uma grande área superficial de contato com a matriz, o que acarreta ligações mais fortes e em maior número, possibilitando que ocorra um completo sinergismo entre matriz e reforço ${ }^{[2,3]}$.

\section{Análise de FTIR}

A Figura 2 mostra os espectros de FTIR para o HDL, poliestireno puro e nanocompósitos. Esta análise é fundamentada na medida de energia absorvida pela vibração de cada uma das ligações químicas presentes nos materiais analisados. Através desta medida de energia absorvida pela ligação química, são caracterizados os materiais sintetizados, determinando e identificando os principais grupos funcionais existentes em suas moléculas.

No espectro do HDL foram verificados valores em números de ondas próximos de 3500 e $1623 \mathrm{~cm}^{-1}$, que correspondem às bandas de deformação axial e angular das moléculas de água respectivamente, presente em seus espaços interlamelares. Na região de $823-425 \mathrm{~cm}^{-1}$ são observadas bandas de vibrações referentes aos $\mathrm{M}-\mathrm{O}$ ou $\mathrm{O}-\mathrm{M}-\mathrm{O}$ (onde $\mathrm{M}=\mathrm{Zn}, \mathrm{Al}$ ) caracterizando as ligações entre os cátions e oxigênio.

São caracterizadas também neste espectro bandas que fazem referência ao perfil do ânion DDS no HDL. Em 2917 e 2850 cm$^{-1}$

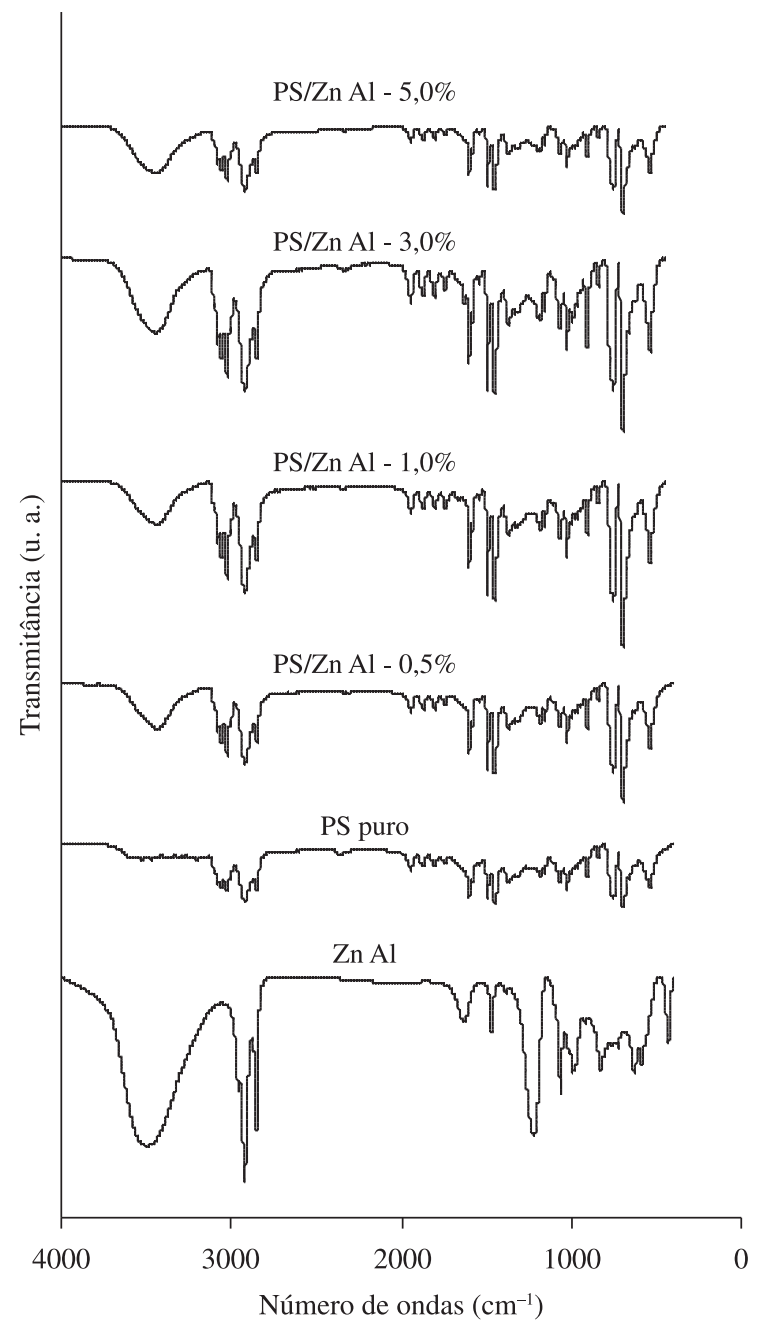

Figura 2. Espectros das análises de FTIR para o HDL (ZnAl), PS puro e nanocompósitos formados por PS/ ZnAl.

são observadas bandas estreitas de deformação axial dos grupos $\mathrm{CH}_{2}$ e $\mathrm{CH}_{3}$ do DDS. Em $1210 \mathrm{~cm}^{-1}$ temos uma banda referente a deformação angular do grupo sulfato também presente no DDS, junto a várias vibrações relativas aos movimentos de deformação da cadeia de $\mathrm{CH}_{2}$ abaixo de $1062 \mathrm{~cm}^{-1}$. Estas bandas relativas ao DDS comprovam mais uma vez que o HDL sintetizado realmente foi modificado.

No espectro do poliestireno puro são observadas bandas de absorção na região de $3068-2847 \mathrm{~cm}^{-1}$ características da deformação axial do grupo C-H. Na região entre $2000-1680 \mathrm{~cm}^{-1}$ são encontradas bandas referentes às ligações (combinações) presentes no anel aromático e em 1598 e $1491 \mathrm{~cm}^{-1}$ bandas da deformação axial do grupo $\mathrm{C}=\mathrm{C}$.

Outras bandas encontradas no espectro do poliestireno puro são na região de 1459 e $1361 \mathrm{~cm}^{-1}$, que caracterizam a deformação angular do grupo $\mathrm{CH}_{2}$, em 747 e $693 \mathrm{~cm}^{-1}$, que caracterizam deformação angular do grupo $\mathrm{CH}$ no anel aromático e em $533 \mathrm{~cm}^{-1}$ referente a deformação axial também do grupo $\mathrm{CH}$ no anel aromático.

Nos espectros dos nanocompósitos é observada uma banda bem característica que existe no HDL e não existe no poliestireno puro em $3500 \mathrm{~cm}^{-1}$, a qual correspondente à deformação axial da 
molécula de água. Também são observadas nestes espectros bandas bem características do poliestireno puro e que não estão presentes no espectro do HDL como em $3068 \mathrm{~cm}^{-1}$, banda característica do grupo C-H e em 2000-1680 cm-1 , que são bandas características das ligações (combinações) encontradas no anel aromático.

Assim, através dos espectros, é possível verificar que os nanocompósitos sintetizados apresentam combinações do HDL e poliestireno, fato que comprova a existência destes dois materiais nos nanocompósitos sintetizados.

\section{Análise de MET}

Na microscopia eletrônica de transmissão (MET) é possível observar a morfologia ou estrutura do nanocompósito, como o HDL está disperso na matriz polimérica, através de imagens.

A Figura 3 possibilita a visualização da análise de MET para o nanocompósito sintetizado com maior percentual de HDL, 5\%.

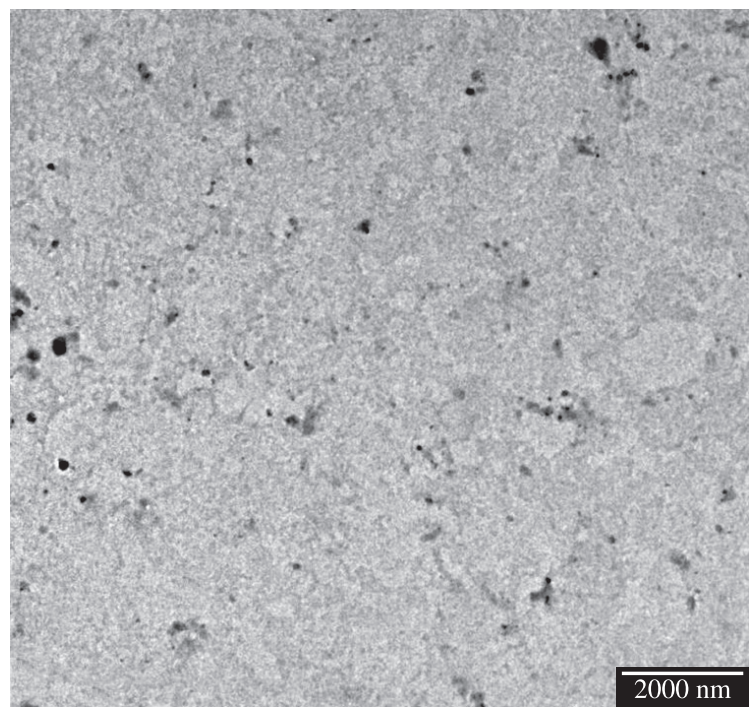

(a)

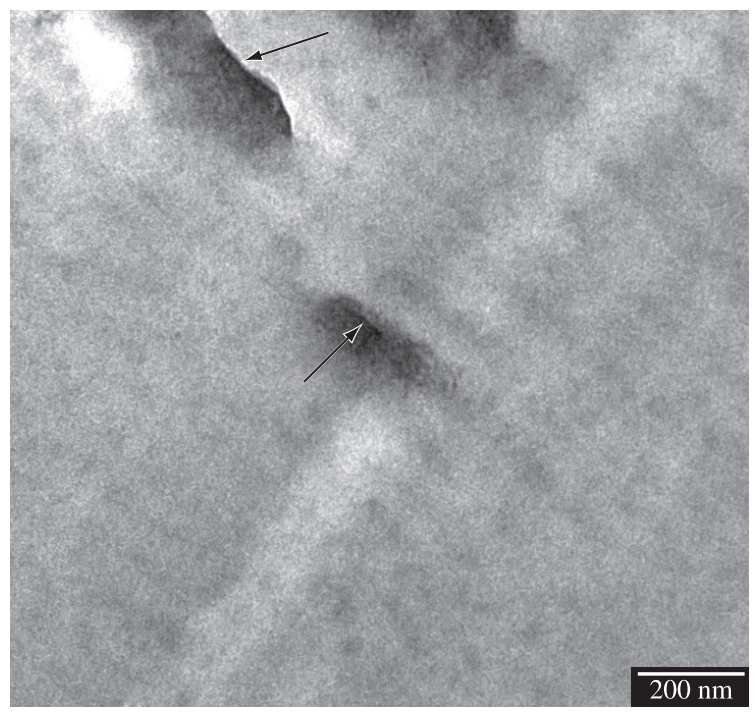

(b)

Figura 3. Imagens da análise de MET para o nanocompósito de PS/ ZnAl $5 \%$. Imagem (a) em baixa resolução e (b) em alta resolução.
Através da Figura 3a é possível observar que as lamelas de HDL, pontos escuros na imagem, estão distribuídas aleatoriamente na matriz polimérica (parte clara da imagem). Não existe um ordenamento das lamelas, estas se apresentam delaminadas, confirmando o resultado obtido na DRX.

$\mathrm{Na}$ Figura $3 \mathrm{~b}$ é apresentada uma imagem do MET em alta resolução. Nesta imagem é possível observar claramente a existência de duas lamelas de HDL, sinalizadas na imagem por setas. Estas lamelas se encontram dispersas na matriz polimérica, não existe evidência destas lamelas apresentarem alguma estrutura cristalina (empilhamento), algum tipo de ordenação, caracterizando a estrutura de natureza esfoliada deste nanocompósito sintetizado. Através desta imagem também é possível estimar a espessura da lamela de HDL dispersa na matriz polimérica, estas lamelas apresentam valores estimados inferiores a $1 \mathrm{~nm}$, valor que corresponde ao apresentado em literatura ${ }^{[10-13]}$. Resultados similares podem ser observados em estudos de nanocompósitos poliméricos e HDL ${ }^{[15-18]}$.

\section{Análise de TGA}

Para a caracterização térmica destes nanocompósitos foi realizada a análise termogravimétrica (TGA). Neste trabalho esta técnica foi utilizada com o objetivo de verificar a estabilidade térmica dos nanocompósitos produzidos e compará-los com o poliestireno puro.

O poliestireno puro apresentou característica muito marcante neste ensaio, como apresenta a Figura 4. Este material começou a se degradar, perder massa, a uma temperatura em torno $360{ }^{\circ} \mathrm{C}$. A partir deste ponto o material polimérico e suas cadeias foram degradadas até valores de temperatura em torno de $427^{\circ} \mathrm{C}$, onde quase a totalidade do material foi consumido.

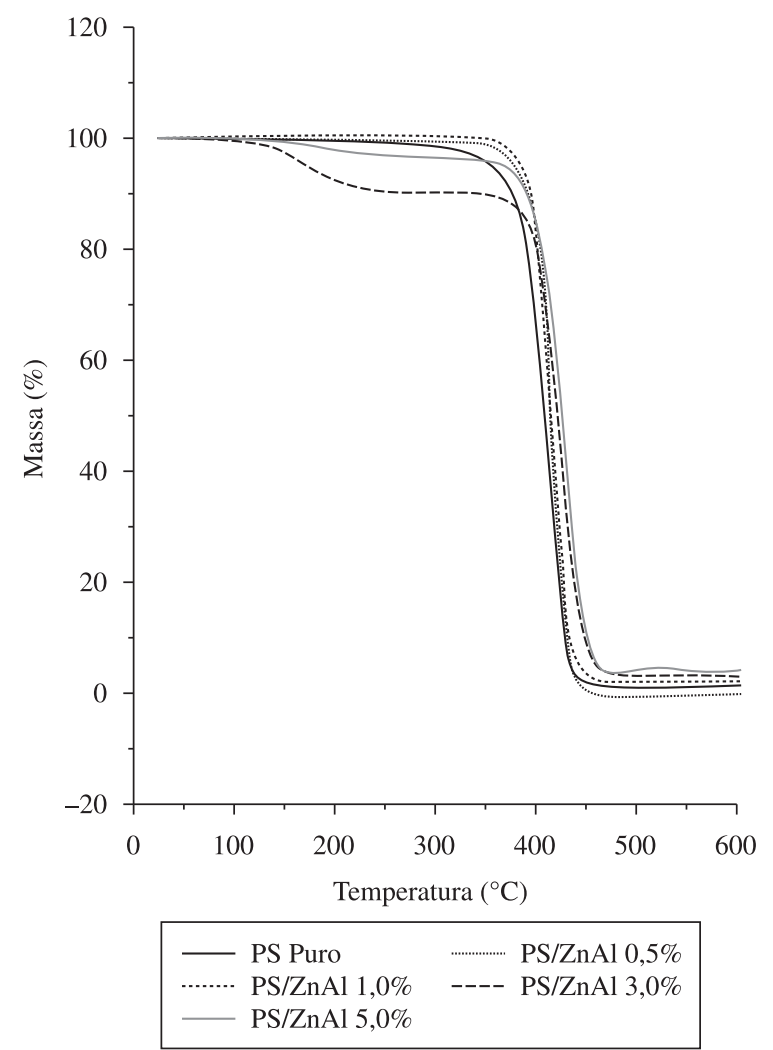

Figura 4. Medidas de TGA para o poliestireno puro e os nanocompósitos de PS - ZnAl. 
Quando os nanocompósitos têm seu comportamento comparado ao do poliestireno, é possível observar que a degradação destes ocorre de forma mais lenta em todos os casos. Basicamente este fato se deve à interação entre o polímero e as lamelas do HDL, onde temos monolamelas com dimensões nanométricas criando uma grande área superficial de contato entre HDL e poliestireno, o que acarreta a formação de ligações mais fortes e em maior número, possibilitando que ocorra um completo sinergismo entre matriz e reforço. Com esta interação entre reforço e matriz, este material apresenta uma maior resistência à difusão de oxigênio e compostos voláteis, outro fato de extrema importância para degradação mais lenta $^{[16-19]}$.

Na análise de TGA para os nanocompósitos é possível verificar um primeiro passo de degradação com valores de 130 a $220{ }^{\circ} \mathrm{C}$, principalmente nos nanocompósitos com 3 e $5 \%$ de HDL, os com 0,5 e $1 \%$ essa degradação é muito sutil. Esta degradação inicial é caracterizada pela evaporação de água presente nos espaços interlamelares e das hidroxilas presentes nas lamelas do HDL. O segundo passo de degradação ocorre em valores de $350{ }^{\circ} \mathrm{C}$ e se estende até a quase completa degradação do material em torno de $450{ }^{\circ} \mathrm{C}$. Este segundo passo corresponde a degradação térmica das cadeias poliméricas presentes no nanocompósito.

Quando utiliza-se $50 \%$ de perda de massa como ponto de comparação entre os materiais são obtidos resultados promissores.

Para a análise realizada no poliestireno puro no ponto de comparação, foi obtido um valor de $410{ }^{\circ} \mathrm{C}$. Neste mesmo ponto de comparação para os nanocompósitos de PS/ ZnAl com 0,5\% foi obtido um valor de $420{ }^{\circ} \mathrm{C}$, com $1 \% 425{ }^{\circ} \mathrm{C}$, com $3 \% 430{ }^{\circ} \mathrm{C}$ e com $5 \% 435{ }^{\circ} \mathrm{C}$. Assim os nanocompósitos com $0,5,1,3$ e $5 \%$ apresentaram um aumento de $10,15,20$ e $25^{\circ} \mathrm{C}$ respectivamente, na temperatura na qual $50 \%$ da massa do material foi perdida. Portanto estes novos materiais apresentam uma maior estabilidade térmica que o poliestireno puro e um promissor futuro para diversas aplicações no campo dos materiais poliméricos.

\section{Conclusão}

Neste trabalho foram sintetizados nanocompósitos de PS/ HDL modificados com DDS. Ensaios de DRX e MET mostraram que os nanocompósitos sintetizados apresentaram estrutura ou morfologia que sugere uma esfoliação e o FTIR permitiu a identificação dos grupos funcionais presentes nos nanocompósitos, comprovando a existência de PS e HDL modificado nestes nanocompósitos.

Os nanocompósitos apresentaram, em todas as composições sintetizadas, estabilidade térmica maior que a do poliestireno puro. Para o nanocompósito com maior quantidade de HDL na composição (5\%), foi obtido um aumento de $25{ }^{\circ} \mathrm{C}$ quando utiliza-se $50 \%$ de perda de peso como ponto de comparação.

Esta maior estabilidade térmica observada nos nanocompósitos sintetizados possibilita um futuro promissor para estes materiais através de uma vasta gama de aplicação destes em diversos campos da indústria e pesquisa.

\section{Agradecimentos}

À CAPES pelo apoio financeiro, ao Laboratório Nacional de Luz Síncotron (LNLS) pelo apoio ao projeto disponibilizando a sua infra-estrutura laboratorial para realização dos ensaios e a Rafael Marangoni pelo conhecimento compartilhado.

\section{Referências Bibliográficas}

1. Esteves, A. C. C.; Timmons, A. B. \& Trindade, T. - Quím. Nova, 27, p.795 (2004).

2. Wang, L.; Su, S.; Chen, D. \& Wilkie, C. A. - Polym. Degrad. Stab., 94, p.770 (2009).

3. Wing Mai, Y.; Zhen Yu, Z. - "Polymer nanocomposites", CRC press LLC, New York (2006).

4. Okada, A.; Kawasumi, M.; Usuki, A.; Kojima, Y.; Kurauchi, T. \& Kamigaito, O. - "Synthesis and properties of nylon-6/clay hybrids", in: Polymer based molecular composites", cap.1, Schaefer D. W. \& Mark, J. E. (ed.), Pittsburgh (1990).

5. Liu, Z.; Ma, R.; Ebina, Y.; Iyi, N.; Takada, K. \& Sasaki, T. - Langmuir., 23, p.861 (2007).

6. Liu, Z.; Ma, R.; Osada, M.; Iyi, N.; Ebina, Y.; Takada, K. \& Sasaki, T. - J. Am. Chem. Soc., 128, p.4872 (2006).

7. Leroux, F.; Meddar, L.; Mailhot, B.; Morlat-Thérias, S. \& Gardette, J. L. - Polymer, 46, p.3571 (2005).

8. Sahu, B. \& Pugazhenthi, G. - J. Polym. Mater., 25, p.407 (2008).

9. Wypych, F. \& Satyanarayana, K. G. - J. Colloid Interface Sci., 285, p.532 (2005)

10. Demir, M. M.; Memesa, M.; Castignolles, P. \& Wegner, G. - Macromol. Rapid. Comm., 27, p.763 (2006).

11. Cavani, F.; Trifirò, F. \& Vaccari, A. - Catal. Today., 11, p.173 (1991).

12. Nogueira, T. R.; Lona, L. M. F.; Mcmanus, N. T.; Vivaldo-Lima, E. \& Penlidis, A. - J. Mater. Sci., 45, p.1878 (2010).

13. Del Arco, M.; Fernándes, A.; Martín, C. \& Rives, C. - Appl. Clay Sci., 36, p.133 (2007).

14. Velu, S.; Suzuki, K.; Okasaki, M.; Osaki, T.; Tomura, S. \& Ohashi, F. Chem. Mater., 11, p.2163 (1999).

15. Qiu, L.; Chen, W. \& Qu, B. - Coll. Polym. Sci., 283, p.1241 (2005).

16. Nyambo, C.; Songtipya, P.; Manias, E.; Jimenez-Gascoc, M. M. \& Wilkie, C. A. - J. Mater. Chem., 18, p.4827 (2008).

17. Ding, P.; Qu, B. - J. Colloid Interface Sci., 291, p.13 (2005).

18. Qiu, L.; Chen, W., Qu, B. - Polym. Degrad. Stab., 87, p.433 (2005).

19. Manzi-Nshuti, C.; Chen, D.; Wilkie, C. A. - Polym. Degrad. Stab., 94, p.1290 (2009).

Enviado: $12 / 01 / 10$

Reenviado: 07/04/10

Aceito: $23 / 07 / 10$

DOI: $10.1590 / \mathrm{S} 0104-14282011005000017$ 